\title{
STRUCTURAL CHANGES OF THE RAT CHOROID PLEXUS FOLLOWING INTRAVENTRICULAR INJECTION OF SOME DRUGS
}

\author{
TRANSMISSION AND SCANNING ELECTRON \\ MICROSCOPIC OBSERVATION
}

\author{
TATSUO SHIMADA, TETSUO INOKUCHI, IKUO ŌBAYASHI \\ AND MASAHIRO MURAKAMI \\ Second Department of Anatomy, Kurume University School of Medicine, \\ Kurume, 830, Japan
}

(Received for publication January 12, 1977)

\begin{abstract}
Ultrastructures of the choroid epithelium of the rats were examined by means of transmission and scanning electron microscopy after intraventricular injection of pilocarpine and puromycin.

In the rats to which isotonic saline solution was injected, peculiar changes were not observed as composed with the rats without drug treatments. Pilocarpine injection produced structural changes suggestive of hyperfunction of the choroid cells. Their nuclei were oval in shape and the cytoplasmic organelles were extensively well-developed. Moreover, these cells possessed polypoidal microvilli with remakably expanded tips. On the other hand, puromycin injection caused structural changes suggesting degeneration or hypofunction of the choroid cells. Their nuclei showed irregular contour and the organelles were also depressed. The microvilli tended to have slender finger-like profile.

In conclusion, the polypoid border which has been know as a characteristic of the choroid epithelium is not artifact in preparations but may be probably concerned with hyperfunction of this tissue.
\end{abstract}

\section{INTRODUCTION}

It has been well known that the free surface of the choroid epithelium is covered with so called "polypoid border" which is microvilli in nature. These microvilli are slightly different in appearance from those of the epithelial cells of the small intestine or the renal proximal tubule. The microvilli observed in the choroid plexus were somewhat expanded at the tip. (Maxwell and Pease, 1956 ; Santolaya and Echandia, 1968 ;
Yamadori, 1972). Generally, the microvilli with expanded tips were regarded as artifacts (Burgos, 1964), but it remains obscure whether the microvilli with expanded tips in the choroid epithelium are artifacts or are related to the activity of cellular function. In order to clarify this problem, we injected pilocarpine and puromycin into the cerebral ventricle of the rats and investigated sequential ultrastructural changes of the choroid epithelium with transmission and scanning electron 
microscope.

In this paper, the fine structure of the microvilli of the choroid epithelium in rats will be mainly discussed.

\section{MATERIALS AND METHODS}

Male adult Wistar rats weighing 150-180 g were used. After anesthesia isozole, they were fixed on the stereotaxic apparatus for rats. The frontal bone and the dura were trepanned, and the lateral ventricle was stereotaxically cannulated with a microtube through which drug administration was performed. Solutions injected were as follows; 1 . physiological saline $(0.2 \mathrm{ml})$ as control, 2. pilocarpine $(0.1 \mathrm{mg}-0.2$ $\mathrm{mg}$ in $0.2 \mathrm{ml}$ physiological saline), and 3. puromycin $(0.5-1.0 \mathrm{mg}$ in $0.2 \mathrm{ml}$ physiological saline). Animals were killed by decapitation 6 hours after injection of the drugs, and the choroid plexus of the lateral ventricle was partially removed.

For transmission electron microscopy (TEM), the tissues were fixed by immersion in cacodylate-buffered glutaraldehyde-formaldehyde mixture ( $\mathrm{pH}$ 7.2) and postfixed with buffered $2 \%$ osmium tetroxide. Then, they were dehydrated in aceton, embedded in Epon 812, and sectioned with an ultramicrotome. Ultrathin sections were routinely stained with uranyl acetate and lead citrate, and examined in HU-12 AS electron microscope.

For scanning electron microscopy (SEM), the tissues were fixed with the same fixative as described above after washing with saline solution. The fixed specimens were dehydrated in aceton, and then dried by critical point drying method (Anderson, 1951). Follwing carbon and Gold-palladium coating, the samples were studied in HFS-II scanning electron microscope.

\section{RESULTS}

\section{Saline-injected controls}

The choroid plexus cells of the control animals injected physiological saline the cerebral ventricle showed no remarkable changes in appearance and structure when compared with the plexus cells of the normal rats as reported previously; the epithelial cells possessed oval nuclei, rod-like or spherical mitochondria, moderately developed Golgi apparatus, and numerous microvilli with somewhat expanded tips.

\section{Pilocarpine-injected animals}

The oral cavity of the animal was filled with saliva upon injecting the drug into the cerebral ventricle. This is due to excessive salivary secretion by pilocarpine injection.

In the TEM figures, the epithelial cells of the chorid plexus were cuboidal in shape and the nuclei were oval. In their cytoplasm, there were abundant polysomes and endoplasmic reticulum, numerous mitochondria, and well-developed Golgi apparatus. These findings may suggest hyperfunction of the choroid plexus cells. Microvilli of these cells possessed remarkably expanded tips (Fig. 1).

In the SEM micrographs, the free surface of the epithelial cells covered by numerous microvilli whose tips were mostly bulbous in shape (Fig. 2). These microvilli were bigger in size than those of the control rats.

\section{Puromycin-injected animals}

The TEM observation revealed that most of the epithelial cells were reduced in volume and became electron dense. The nucleus showed an irregular contour. In the cytoplasm, the number of 
polysomes as well as endoplasmic reticulum was decreased, and Golgi apparatus was also constricted. In addition, the majority of microvilli became slender in shape (Fig. 3). These findings may suggest hypofunction or degeneration of the choroid epithelium.

The SEM analysis indicated that the microvilli of the epithelial cells of the choroid plexus had finger-like profile. Each microvillus was about $0.10-0.15 \mu$ in diameter and smaller as compared with that of the control or pilocarpineinjected rats (Fig. 4).

\section{DISCUSSION}

Maxwell and Pease (1956) reported that the ventricular surface of the choroid epithelium in mammals was covered with numerous microvilli, which were characterized by polypoid formation, and they assumed that the microvilli actually pinch off and become a part of the secretion. However, recent reports demonstrated that the microvilli of the choroid epithelium were various in shape such as filiform, clavate or polypoid (Santolaya and Echandia, 1968; Dohrmann and Bucy, 1970 ; Yamadori, 1972). Althouth, the functional significance of these microvilli remained still obscure.

In the present study, the majority of the choroid cells seen in the pilocarpine-injected rats showed remarkable polypoid formation. Further, the nucleus was enlarged and cytoplasmic organelles were extensively well-developed as compared with the controle. These structural features were evidently indicative of hyperfunction of the choroid cells. This ultrastructural change may be consistent with predencessor's reports that pilocarpine-injection has stimulated secretion of cerebrospinal fluid (CSF) (Wislocki, 1932; Santolaya and Echandia, 1968 ; Colins and Morris,
1975), while, administration of acetazolamide which is used clinically to lower the secretion rate of $\mathrm{CSF}$ has caused a significant reduction in number of the choroid cells with enlarged microvilli (Colins and Morris, 1975).

Accordingly, it may be presumed that the microvilli with expanded tips have reference to active secretion of CSF.

On the other hand, after intraventricular injection of puromycin which has been known to inhibit protein synthesis (Gambetti et al., 1968), the choroid cells became atropic: both nucleus and cytoplasm were reduced in volume, the cytoplasmic organelles were also depressed. In addition, it would be worthwhile to note that the microvilli of these cells not polypoid but slender, finger-like appearance. These ultrastructural changes in puromycin-injected rats may be probably related to degeneration or hypofunction of the choroid cells.

\section{REFERENCES}

Anderson, T. F. (1951). Techniques for the preservation of three-dimensional structure in preparing specimens for the electron microscope. Trans. N. Y. Acad. Sci., 13, 130-134.

Burgos, M.H. (1964). Uptake of colloidal particles by cells of the epididymis. Anat. Rec., 148, 517-525.

Collins, P. and Morriss, G. M. (1975). Changes in the surface features of choroid plexus of the rat following the administration of acetazolamide and other drugs which affect CSF secretion. J. Anat., 120, 571-579.

Dohrmann, G. J. and Bucy,P.C. (1970). Human choroid plexus: a light and eletron microscopic study. J. Neurosurg., 33, 506-516.

Gambetti, P., Gonatas, N. K. and Flexner, L. B. (1968). The fine structure of puromycin-induced changes in mouse entorhinal cortex. J. Cell Biol., 36, 379-390.

Maxwell, D. S. and Pease, D. C. (1956). The electron microscopy of the choroid plexus. J. biophy. biochem. Cytol., 2, 467-474. 
Santolaya, R. C. and Echandia, E. L. R. (1968). The surface of the choroid plexus cell under and experimental conditions. Z. Zellforsch. 92, 43-51.

WISLOCKI, G. B. (1932). The cytology of the cerebrospinal pathway. In: Special Cytology, 3, 1485-1521. Hafser Publ. Co., New York.

YAMADORI, T. (1972). A scanning electron microscopic observation of the choroid plexus in rats. Arch. hitol. jap., 35, 89-97.

\section{EXPLANATION OF FIGURES}

Fig. 1 Transmission electron micrograph of the choroid plexus in a pilocarpine-injected rat. The choroid cell posseses numerous microvilli (MV) with expanded tips. $\times 18,000$

Fig. 2 Scanning electron micrograph of the free surface of the choroid plexus in a pilocarpine-injected rat. The tips of microvilli show spherical appearance. $\times 14,000$

Fig. 3 Transmission electron micrograph of the choroid plexus in a puromycin-injected rat. Many slender microvilli (MV) are protruding from the apical end of the choroid cell. $\times 20,000$

Fig. 4 Scanning electron micrograph of the free surface of the choroid plexus in a puromycin-injected rat. The microvilli show slender finger-like profile. $\times 13,000$ 

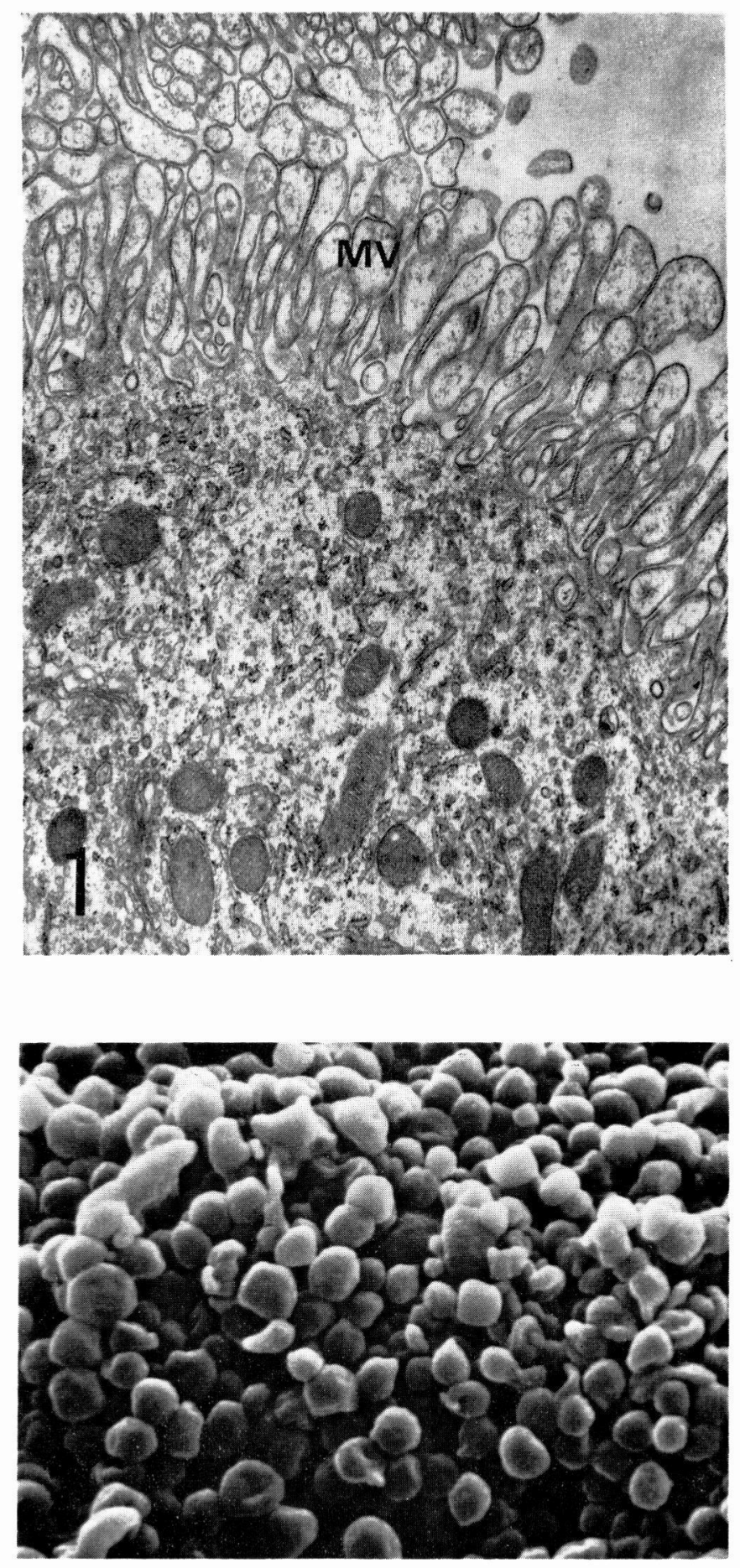

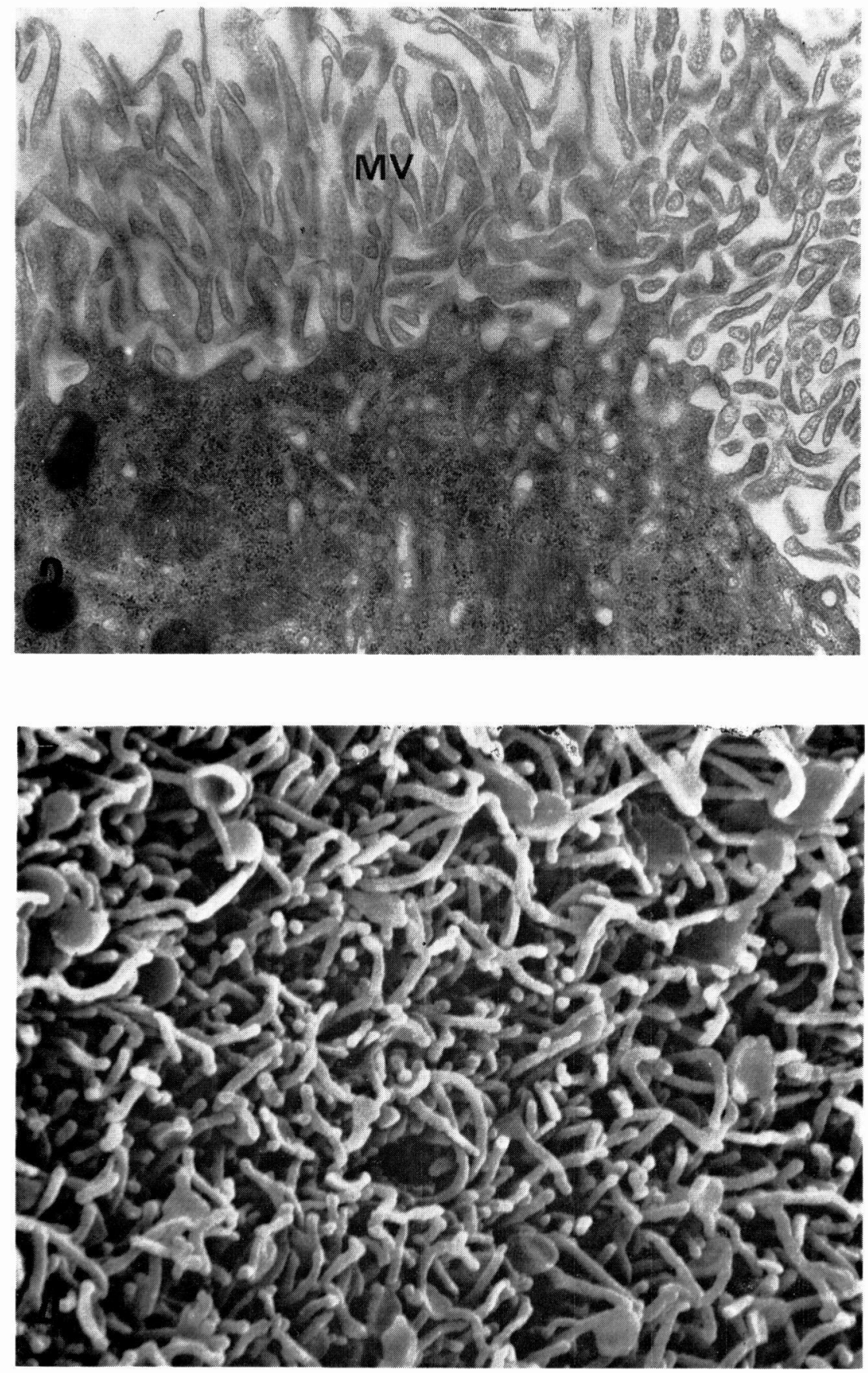